\title{
Self-Assembly of Graphene Single Crystals with Uniform Size and Orientation: the First 2D Super-Ordered Structure
}

\author{
Mengqi Zeng, ${ }^{, \dagger}$ Lingxiang Wang, ${ }^{\S, \dagger}$ Jinxin Liu, ${ }^{\dagger}$ Tao Zhang, ${ }^{\dagger}$ Haifeng Xue, ${ }^{\dagger}$ Yao Xiao, ${ }^{\dagger}$ Zhihui Qin*, ${ }^{*}$ and Lei \\ $\mathrm{Fu}^{* \dagger}$ \\ ${ }^{\dagger}$ College of Chemistry and Molecular Science, Wuhan University, Wuhan 430072, P. R. China \\ ${ }^{\star}$ State Key Laboratory of Magnetic Resonance and Atomic and Molecular Physics, Wuhan Institute of \\ Physics and Mathematics, Chinese Academy Sciences, Wuhan 430071, P. R. China \\ ${ }^{\S}$ These authors contributed equally to this work. \\ *Towhom correspondence should be addressed: email: leifu@whu.edu.cn, zhqin@wipm.ac.cn
}




\section{Experiments and characterizations}

CVD growth of graphene super-ordered structure (GSOS): $50 \mu \mathrm{m}$ thick $\mathrm{Cu}$ foils (99.8\% purity) and $100 \mu \mathrm{m}$ thick W foils (99.95\%) were purchased from Alfa Aesar. Poly (methyl methacrylate) (PMMA) was used as the solid carbon source, which was spin-coated on the $\mathrm{Cu}$ foil, and was dried at $120{ }^{\circ} \mathrm{C}$ for $30 \mathrm{~min}$. Then the $\mathrm{Cu}$ foil was directly placed onto a $\mathrm{W}$ foil of the same size. CVD growth of the graphene was conducted in a thermal tube furnace (HTF 55322C Lindberg/Blue M). The diameter of the quartz tube is $2.5 \mathrm{~cm}$. The sample was rapidly heated to an elevated temperature $\left(1080 \sim 1120^{\circ} \mathrm{C}\right)$ in the atmosphere of $\mathrm{H}_{2}$ with flow rate ranging from 200 to $400 \mathrm{sccm}$ under ambient pressure. After that, the sample was annealed for $2 \mathrm{~min}$ in a gas mixture in which the flow rates of $\mathrm{Ar}$ and $\mathrm{H}_{2}$ were adjusted to the desired values, which was aimed at controlling the arrangement of the seeds by exerting directional disturbance. The Ar flow rate ranges from 400 to $800 \mathrm{sccm}$ and the $\mathrm{H}_{2}$ flow rate ranges from 200 to $400 \mathrm{sccm}$. The following growth of GSOS was typically conducted under $3 \mathrm{sccm} \mathrm{CH}_{4}$ and $300 \mathrm{sccm} \mathrm{H}_{2}$ for 6 min. Finally, the $\mathrm{CH}_{4}$ was turned off, and the system was rapidly cooled to the room temperature.

CVD growth of $\mathrm{MoS}_{2}$ single crystal: $\mathrm{MoO}_{3}$ powder (Alfa Aesar, 99.9995\%, $25 \mathrm{mg}$ ) was placed in the center of the heating zone in the furnace, and a quartz boat filled with sulfur powder (Alfa Aesar, $99.999 \%, 0.5 \mathrm{~g}$ ) was placed in the upstream along with the gas flow direction, $25 \mathrm{~cm}$ away from the $\mathrm{MoO}_{3}$ powder. 300-nm-thick $\mathrm{SiO}_{2} / \mathrm{Si}$ substrates with $\sim 1 \times 1 \mathrm{~cm}^{2}$ size were placed close to the $\mathrm{MoO}_{3}$ powder. The substrates were heated to $650{ }^{\circ} \mathrm{C}$ in 15 minutes with $200 \mathrm{sccm}$ Ar under ambient pressure. When the temperature of the furnace reached at $650^{\circ} \mathrm{C}$, sulfur powder was heated by a heating belt with an individual temperature controller at $150{ }^{\circ} \mathrm{C}$. After $10 \mathrm{~min}$, the furnace was heated up to $850{ }^{\circ} \mathrm{C}$ and kept at this temperature for $1 \mathrm{~h}$. The temperature for vaporizing sulfur powder was maintained at $150{ }^{\circ} \mathrm{C}$ throughout the whole synthesis. After the process, the heating belt was removed away and the furnace was cooled to the room temperature.

Device Fabrication: The GSOS was transferred onto $300 \mathrm{~nm} \mathrm{SiO}_{2} / \mathrm{Si}$ substrates via the PMMA-assisted transfer method. This was followed by acetone rinsing to remove the PMMA residues. Field-effect transistors (FETs) were fabricated via a standard e-beam lithography (EBL) procedure. The electrodes $(10 \mathrm{~nm} \mathrm{Ti} / 50 \mathrm{~nm} \mathrm{Au})$ were deposited by thermal evaporation. The lift-off process was performed with acetone followed by isopropyl alcohol cleaning. The $\mathrm{MoS}_{2}$ single crystals were directly transferred onto the GSOS-based electrode arrays via the PMMA transfer method, in which the PMMA was also removed by acetone rinsing. 
Characterizations: Optical images were taken with an optical microscope (Olympus DX51), and Raman spectroscopy was performed with a laser micro-Raman spectrometer (Renishaw in Via, 532 $\mathrm{nm}$ excitation wavelength). X-ray photoelectron spectroscopy (XPS) was performed on ESCALAB 250Xi using monochromatic Al Ka radiation (225 W, $15 \mathrm{~mA}, 15 \mathrm{kV})$. Scanning electron microscopy (SEM) images were obtained by a ZEISS Merlin Compact SEM. Scanning tunneling microscopy (STM) characterization and low energy electron diffraction (LEED) characterization were performed with an ultrahigh vacuum (less than $1 \times 10^{-10}$ Torr) STM system (Unisoku USM1300S 3He STM, Japan) at low temperature (at liquid nitrogen temperature, $\sim 78 \mathrm{~K}$ ) and at room temperature, respectively. The current (I)-voltage (V) data were collected in a probe station under ambient. 


\section{The self-assembly process of GSOS on liquid $\mathrm{Cu}$}

PMMA is introduced as a solid carbon source to offer the seeds, and it is spin-coated on the $\mathrm{Cu}$ foil. The corresponding SEM image is exhibited in Figure S1a. As the temperature of the furnace increases to the melting point of $\mathrm{Cu}\left(\sim 1080{ }^{\circ} \mathrm{C}\right)$ rapidly, the $\mathrm{Cu}$ foil melts and, meanwhile, PMMA film decomposes and condenses, thus the seeds form. When given appropriate airflow with uniform speed and direction, the original distribution of the seeds will be broken and the seeds tend to arrange in an energetically favorably way on the rheological surface, in which the spacing between the neighboring seeds will be nearly the same for balancing the whole energy on the liquid surface (Figure S1c). Otherwise, without the airflow disturbance, the seeds tend to stay at the original decomposing site and arrange randomly on the liquid $\mathrm{Cu}$ surface (Figure $\mathrm{S} 1 \mathrm{~b}$ ). Then $\mathrm{CH}_{4}$ is introduced for the growth of the seeds. As the growing progresses, the mutual electrostatic force from the adjacent crystals continually increases that ultimately resulted in the formation of GSOS (Figure S1d).

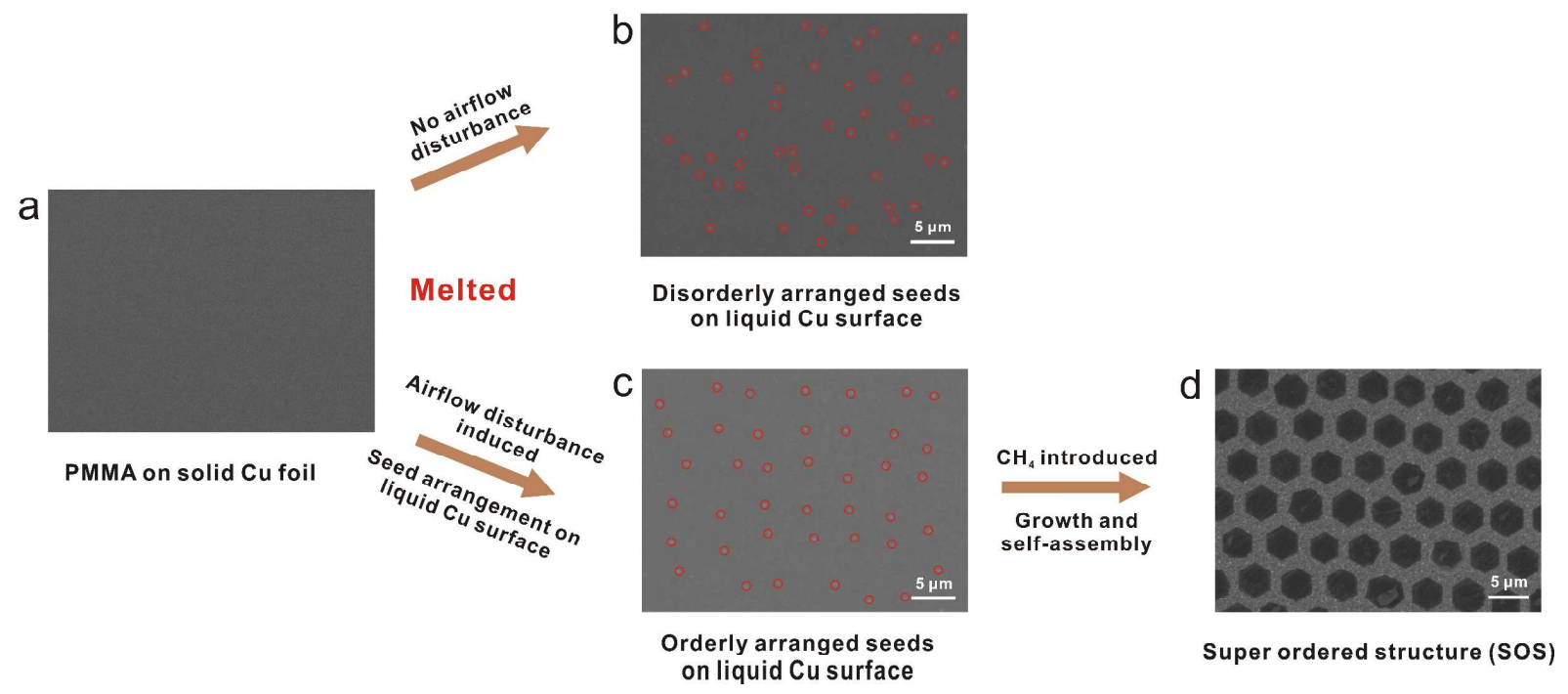

Figure S1. SEM images showing the self-assembly process of GSOS on liquid $\mathrm{Cu}$. (a) SEM image of the PMMA film spin-coated on solid $\mathrm{Cu}$. (b) SEM image showing the formation of disorderly arranged seeds formed via the decomposition and agglomeration of the PMMA after pre-annealing without airflow disturbance. (c) SEM image showing the seed arrangement induced by the airflow disturbance with uniform and steady flow rate and a certain direction. (d) SEM image of the GSOS derived from the seed growth driven by the static controlled self-assembly process. 


\section{Raman spectra of the graphene single crystals in the GSOS}

The uniformity and quality of graphene single crystals in the SOGS were examined by Raman spectra. The statistical study over 100 sampling data collected from randomly selected graphene single crystals suggests that the as-obtained GSOS own extremely high uniformity and quality. Figure S2 shows the selected 20 Raman spectra. The D peaks, indicating defects of graphene, are not obvious, which attests to high crystalline quality. The intensity ratios $\left(\mathrm{I}_{2 \mathrm{D}} / \mathrm{I}_{\mathrm{G}}\right)$ are larger than 2 , indicating that the samples are single-layer.

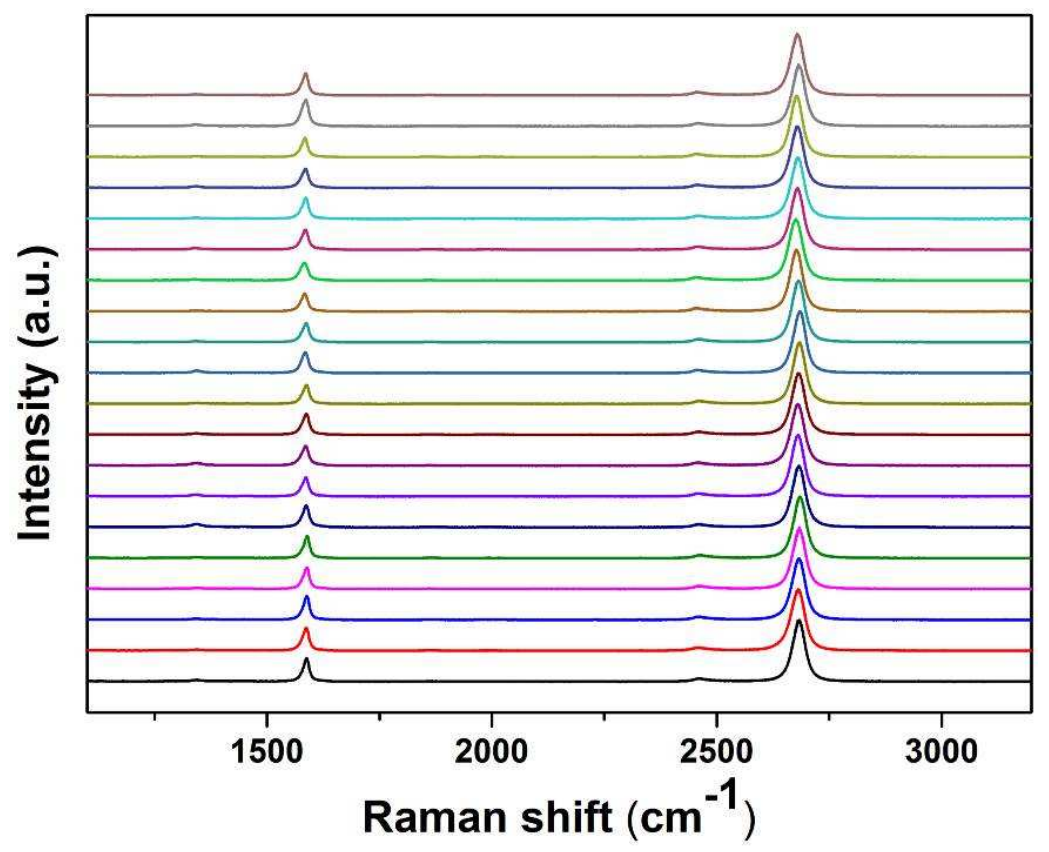

Figure S2. The selected 20 Raman spectra performed from the graphene single crystals in the GSOS. 


\section{STM images of different regions in the GSOS}

For exhibiting the consistent orientation of the graphene single crystals in the GSOS, many STM images were captured at randomly selected positions (Figure S3), which show relatively good uniformity of the crystallographic orientation. The inset in Figure S3e indicates the distribution of the rotation angle. The slight deviation can be contributed to the deformation of the $\mathrm{Cu}$ foil after the annealing process at elevated temperature.

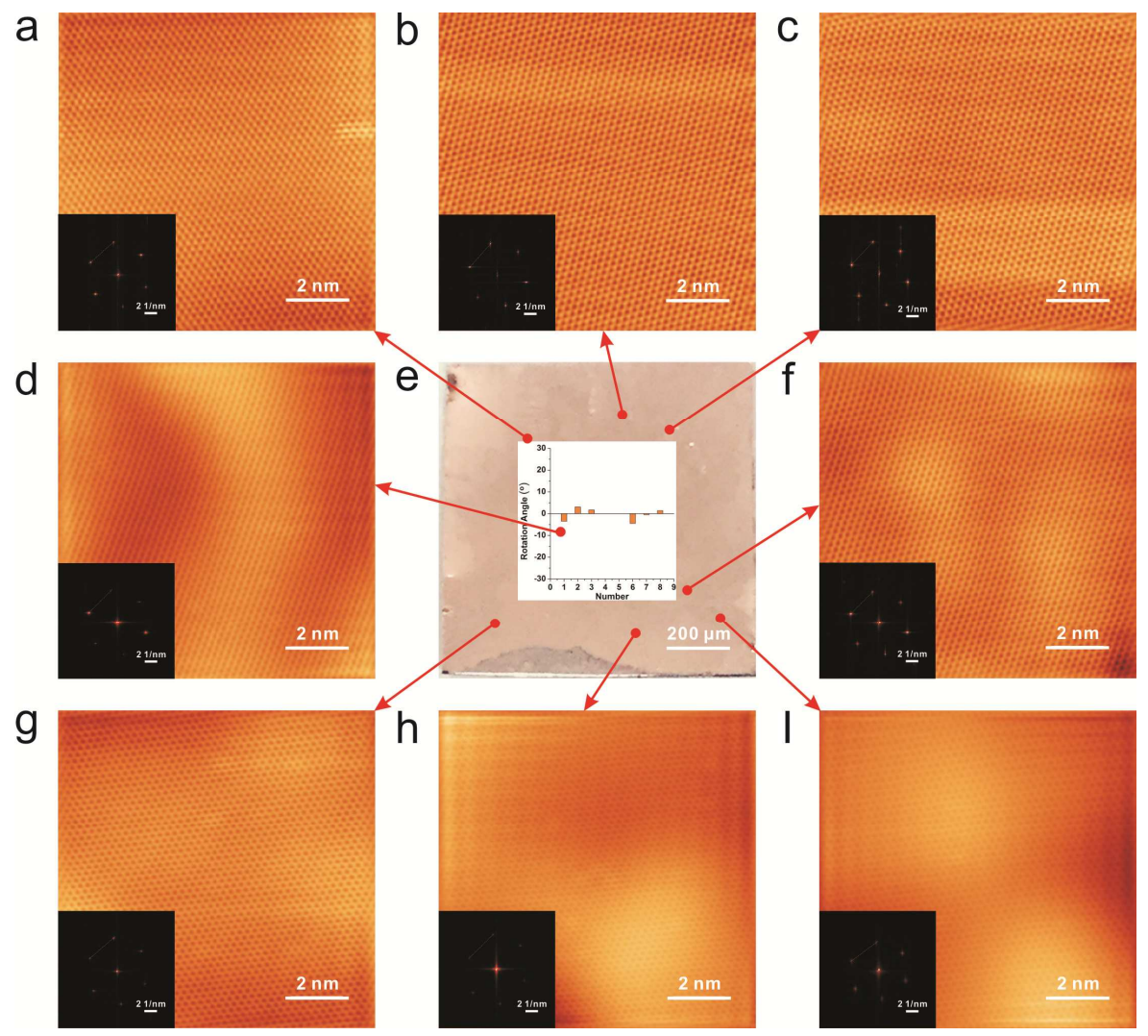

Figure S3. Honeycomb graphene structures and the corresponding 2D fast Fourier transformation (FFT) patterns of 8 regions in the GSOS on $\mathrm{Cu}$ substrate. ${ }^{1}$ The distance between each sampling site is several hundreds of micrometers. The orange dash lines marked in each FFT image were used to show the orientation difference more clearly. The inset in (e) shows the distribution of the rotation angle. 
5. Step-by-step magnified STM images of an individual graphene single crystal in the GSOS

Typical step-by-step magnified STM images were offered to show the characteristic monolayer graphene and clear honeycomb structures.

a
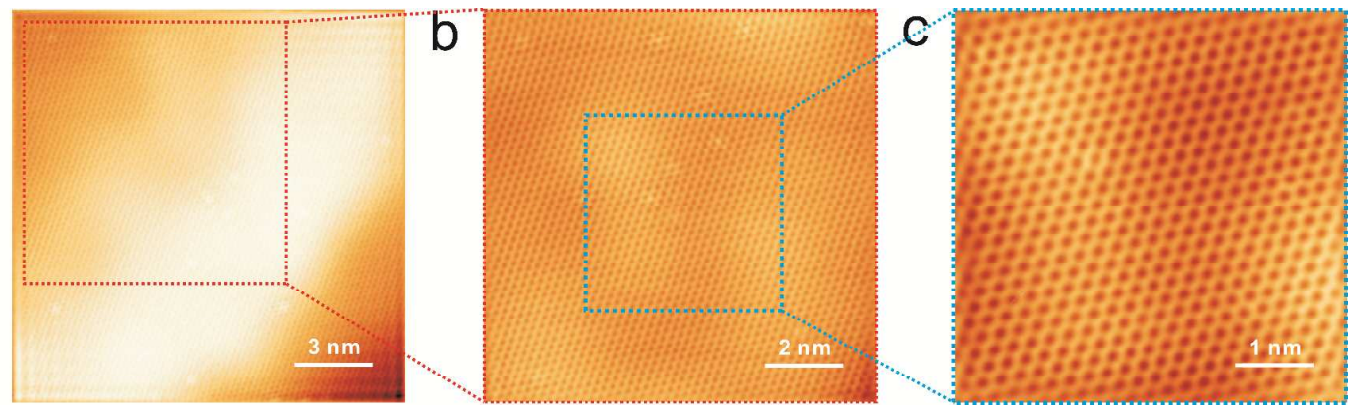

Figure S4. Typical step-by-step magnified STM images of an individual graphene single crystal in the GSOS. 


\section{Various GSOS with different distance and size under different parameters}

The influence of $\mathrm{H}_{2}$ flow rate, the annealing temperature and the annealing time, which were closely related to the process of the seed formation and arrangement and laid the foundation for the assembly of the graphene single crystals, were clarified, to systematically study the impact factors and thus obtained deeper understanding of the formation of GSOS. Here we determine the distance between the centres of two parallel adjacent graphene single crystals as the characteristic distance of GSOS, and the diagonal distance of the hexagonal graphene single crystal as its size.

Firstly, we studied the influence of the $\mathrm{H}_{2}$ and it will have dual functions in the arrangement of the seeds. As an organic polymer, PMMA will be decomposed by the etching effect of $\mathrm{H}_{2}$ under elevated temperature. The larger flow rate of $\mathrm{H}_{2}$ will lead to relatively lower density of seeds, as a result, the distance of each seed would be larger. On the other hand, similar to the Ar flow, the $\mathrm{H}_{2}$ flow rate also takes a part in adjusting the arrangement of the seeds. Corresponding to the $\mathrm{H}_{2}$ flow rate of 200, 300 and $400 \mathrm{sccm}$, the mean distance is 6.26, 7.68 and $6.85 \mu \mathrm{m}$, respectively (Figure S5), in which the distance doesn't change monotonously with the $\mathrm{H}_{2}$ flow rate and is in consistence with the situation for the Ar flow. In addition, the $\mathrm{H}_{2}$ will also have influence on the subsequent graphene growth, since the etching effect will suppress the enlargement of the graphene single crystals. Corresponding to the $\mathrm{H}_{2}$ flow rate of 200, 300 and $400 \mathrm{sccm}$, the derived size of graphene single crystals is 5.20, 5.02 and $4.41 \mu \mathrm{m}$, respectively (Figure S5).
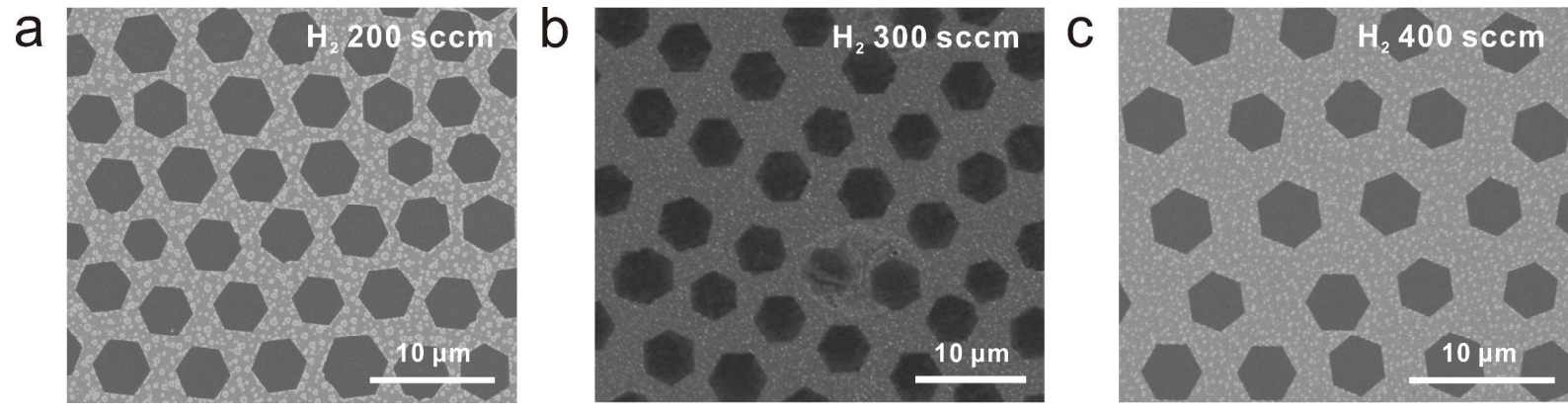

Figure S5. The typical SEM images of the GSOS grown on $\mathrm{Cu}$ under different $\mathrm{H}_{2}$ flow rate. (a) 200 sccm. (b) $300 \mathrm{sccm}$. (c) $400 \mathrm{sccm}$. In addition, the other annealing and growth parameters remained the same. The flow rate of Ar was $600 \mathrm{sccm}$. The annealing process was remained for $2 \mathrm{~min}$ at $1080{ }^{\circ} \mathrm{C}$. The subsequent growth was sustained under $3 \mathrm{sccm} \mathrm{CH}_{4}$ for $6 \mathrm{~min}$.

Secondly, we studied the influence of the annealing time. Long-time exposure under $\mathrm{H}_{2}$ will make the seeds smaller and sparser due to the etching of $\mathrm{H}_{2}$ to PMMA film. Thus, the distance between each graphene single crystal will change with the annealing time. As shown in Figure S6, as 
the annealing time is increased from 2 to $5 \mathrm{~min}$, the distance can be 7.89, 9.53, $10.01 \mu \mathrm{m}$, respectively. In addition, due to the same flow rate of $\mathrm{CH}_{4}$ and $\mathrm{H}_{2}$, and growth time, the mean size of the graphene single crystals could be nearly the same, and was 7.14, 7.06 and $7.20 \mu \mathrm{m}$, respectively.
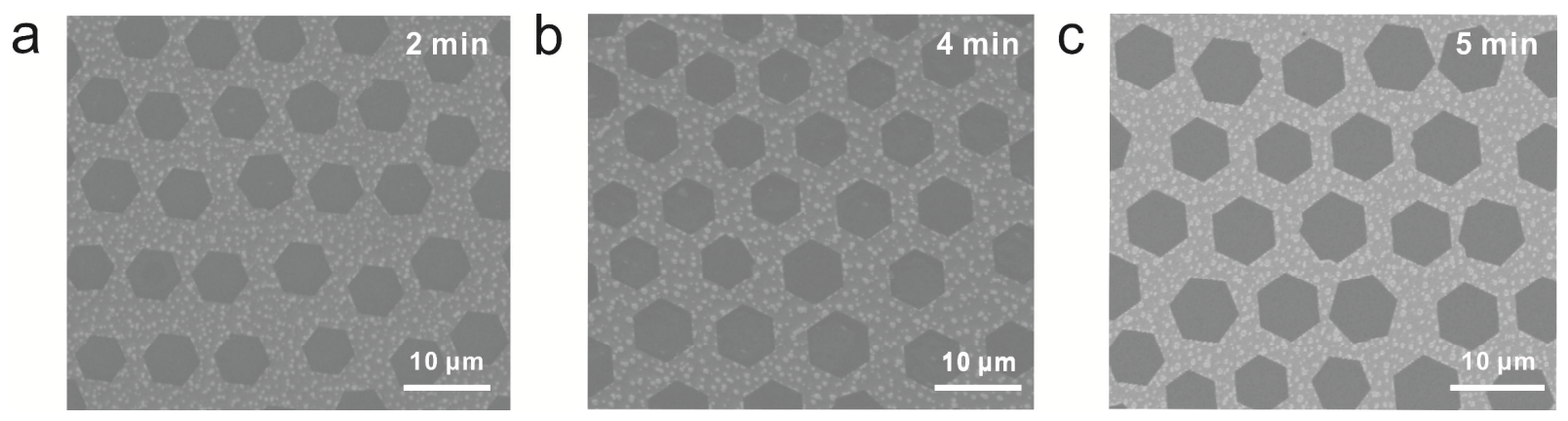

Figure S6. The typical SEM images of the GSOS grown on $\mathrm{Cu}$ under different annealing time. (a) 2 min. (b) 4 min. (c) 5 min. In addition, the other annealing and growth parameters remained the same. The flow rate of Ar was $600 \mathrm{sccm}$ and the flow rate of $\mathrm{H}_{2}$ was $300 \mathrm{sccm}$. The annealing process and growth process was both remained at $1080{ }^{\circ} \mathrm{C}$. The subsequent growth was sustained under $4 \mathrm{sccm}$ $\mathrm{CH}_{4}$ and $300 \mathrm{sccm} \mathrm{H}_{2}$ for 6 min.

Thirdly, we performed the experiment under different annealing and growth temperature. The higher annealing temperature will result in faster etching from $\mathrm{H}_{2}$ to decompose the PMMA. And higher growth temperature will facilitate the growth of graphene single crystals due to the higher catalyzation of liquid $\mathrm{Cu}$ for the $\mathrm{CH}_{4}$ decomposition. Thus, the distance between each seed and the size of the graphene single crystals should increase with the annealing temperature. As shown in Figure S7, with the temperature ranging from 1080 to $1120{ }^{\circ} \mathrm{C}$, the mean distance is $5.78,7.23$ and $11.87 \mu \mathrm{m}$, respectively. And for the size of the single crystal, the value is $5.11,7.10,11.65 \mu \mathrm{m}$, respectively.

a

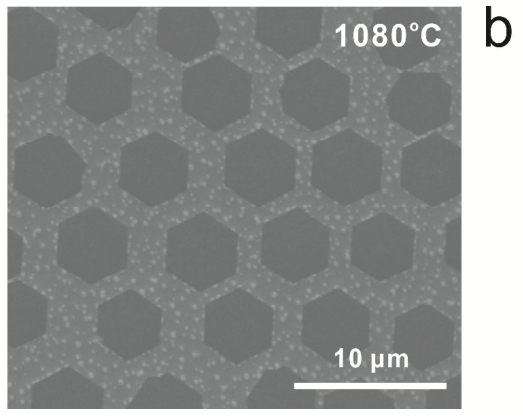

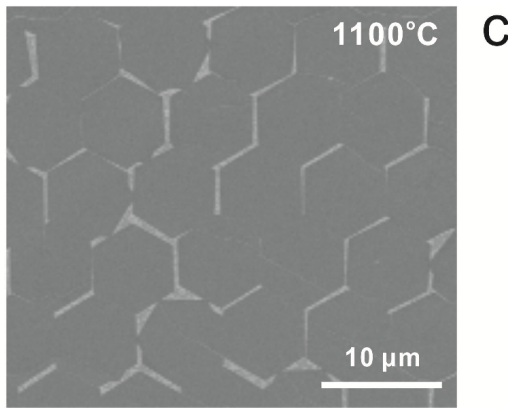

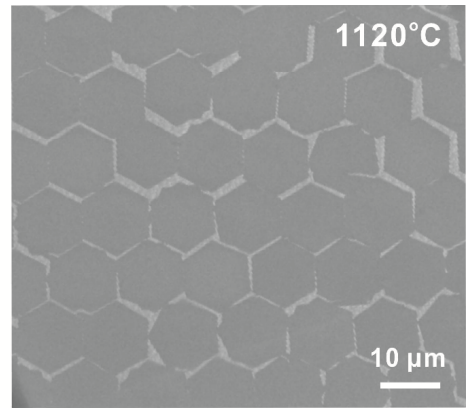

Figure S7. The typical SEM images of the GSOS grown on $\mathrm{Cu}$ under different annealing temperature. (a) $1080{ }^{\circ} \mathrm{C}$. (b) $1100{ }^{\circ} \mathrm{C}$ (c) $1120{ }^{\circ} \mathrm{C}$. In addition, the other annealing and growth parameters remained the same. The flow rate of Ar was $600 \mathrm{sccm}$ and the flow rate of $\mathrm{H}_{2}$ was 200 
sccm. The annealing process was remained for $2 \mathrm{~min}$. The subsequent growth was sustained under 3 sccm $\mathrm{CH}_{4}$ and $200 \mathrm{sccm} \mathrm{H}_{2}$ for 5 min.

In all, the airflow disturbance induced seed arrangement and the subsequent growth conditions determined the characteristic parameters of the as-obtained GSOS. And all the GSOS exhibited excellent uniformity in size and orientation as well as the distance, which confirmed the self-assembly behavior of each graphene single crystal and was ensured by the mutual electrostatic force from the adjacent crystals. 


\section{ESP distribution of the graphene single crystals with different carbon atoms}

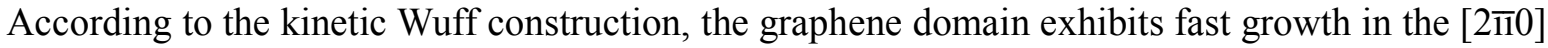
direction and slow growth in the [1010] direction, thus the carbon species favorably combine at the angle of the hexagonal graphene single crystals. ${ }^{2}$ On the other hand, hydrogen-rich atmosphere makes the carbon atoms keep at a hydrogen saturation state. Based on those two presuppositions, we considered that the epitaxial growth of graphene single crystals on liquid $\mathrm{Cu}$ started from several rings of carbon. The $\mathrm{C}$ atoms at the angle of the hexagonal rings will be more reactive than the $\mathrm{C}$ atoms at the edge. The $\mathrm{C}$ atoms at the angle are more inclined to combine with $\mathrm{H}$ atoms, forming active free radicals such as $-\mathrm{CH}$ and $-\mathrm{CH}_{2}$. The Window version of the Gaussian 09 (G09 W) suite of programs was employed in this study. The molecular structures as well as electrostatic potential (ESP) maps are visualized. The PM3 function is used in Semi-empirical studies as it has been found to yield reliable results. ${ }^{3,4}$ ESP maps are obtained by using continuous electronic charge density distribution. The result shows that the edge of the graphene single crystal has an anisotropic electrostatic potential distribution.

Graphene single crystals with different numbers of carbon rings were shown in Figure S8. We took a graphene single crystal with 7 rings as the reference, and proceeded to 21 rings, 37 rings, and 61 rings. The electrostatic potential distribution of the larger ring is more anisotropic, which will facilitate the electrostatic repulse induced self-assembly of the graphene single crystals on the liquid surface.
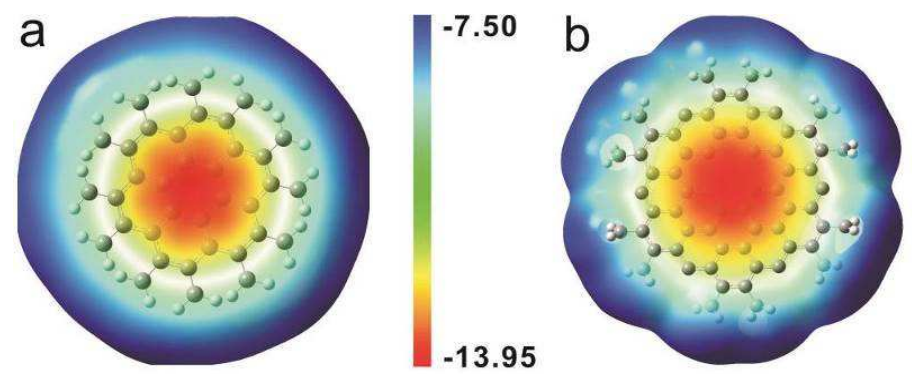

$-11.00$
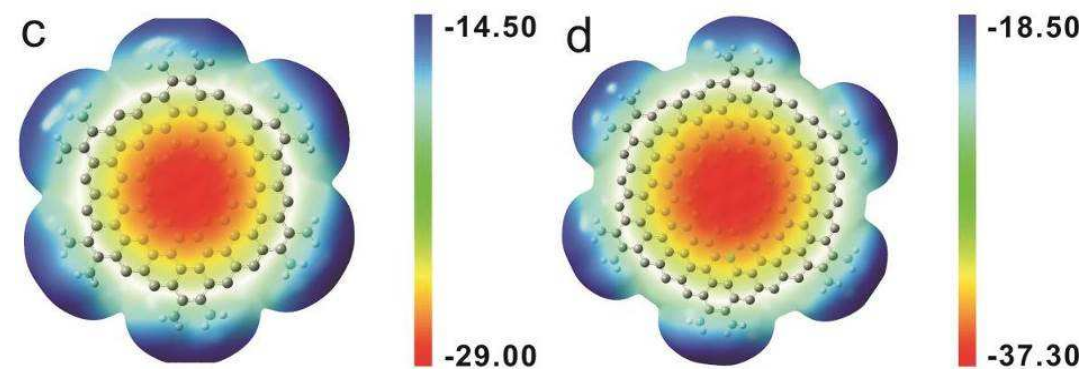

Figure S8. Calculated ESP on the molecular surfaces of graphene single crystals which consist of different number of rings, (a) 7 rings, (b) 21 rings, (c) 37 rings and (d) 61 rings. 


\section{N-doped GSOS}

N-doped GSOS was synthesized via using carbamide to act as the solid carbon source for the formation of the seeds in order to achieve the doping of the GSOS. X-ray photoelectron spectroscopy (XPS) was performed on the N-doped GSOS transferred onto a $300 \mathrm{~nm} \mathrm{SiO}_{2} / \mathrm{Si}$ substrate to detect the N-doping level in graphene (Figure S9). The main C 1s peak located at 284.8 eV corresponds to the graphite-like $\mathrm{sp}^{2} \mathrm{C}$ (Figure S9a). The other three peaks are located at 285.8, 287.1, and $289.0 \mathrm{eV}$, reflecting two different $\mathrm{C}-\mathrm{N}$ bonding structures with $\mathrm{sp}^{2}$ - and $\mathrm{sp}^{3}$ - hybridized carbon and $\mathrm{C}-\mathrm{O}$ bonding structure, respectively. The clear $\mathrm{N} 1 \mathrm{~s}$ peak is observed, confirming the existence of $\mathrm{N}$ (Figure S9b). The asymmetric $\mathrm{N}$ 1s peak can be divided into two components located at 400.7 and $402.6 \mathrm{eV}$, which correspond to graphitic and oxidized nitrogen, respectively. All the peaks were in consistence with the reported work. ${ }^{5}$ The estimation of $\mathrm{N}$ doping level is $1.56 \%$. The SEM image of N-doped GSOS is shown in Figure S9c, which also possess uniform size, spacing and rotation angle. When compared to intrinsic GSOS, the gap among the N-doped graphene grains is significantly decreased, which is shown in Figure S9d. Thus, it is promising to obtain GSOS with adjustable properties.
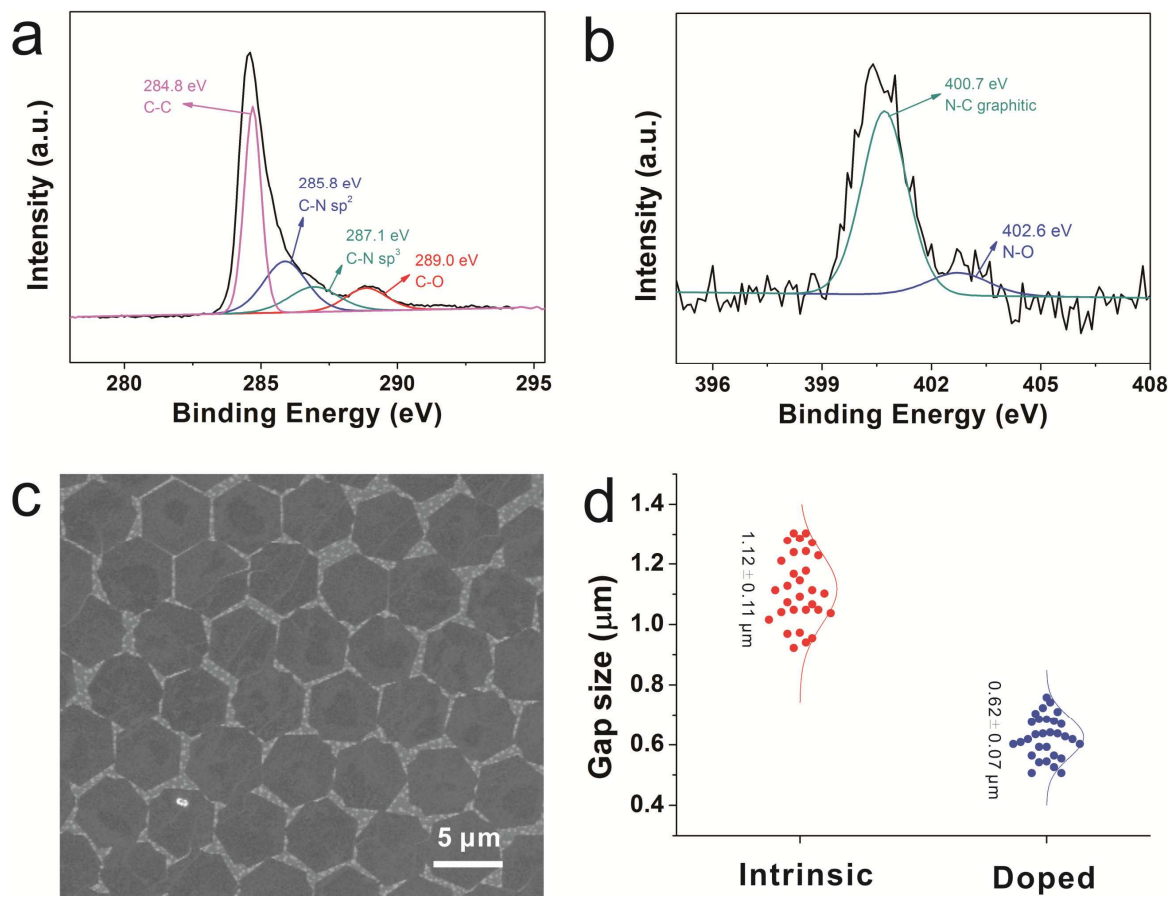

Figure S9. Characterizations of N-doped GSOS. (a) and (b) XPS spectra of N-doped GSOS on Cu substrate. (c) SEM image of N-doped GSOS on solidified $\mathrm{Cu}$ substrate. (d) The statistical distribution about the deviation of gap size of intrinsic and doped GSOS. 


\section{Electrical characteristics of FET devices based on the GSOS}

The electrical performance of thus-fabricated GSOS was examined by constructing back-gated field-effect transistor (FET) arrays. The source/drain (S/D) metal contacts were $10 \mathrm{~nm} \mathrm{Ti} / 50 \mathrm{~nm} \mathrm{Au}$, made by thermal evaporation. Highly-doped $\mathrm{Si}$ substrate with $300 \mathrm{~nm} \mathrm{SiO}$ dielectric layer was used as the back gate. The measurements were performed at room temperature in air. The derived mobilities were found to be ranging from 3000 to $6000 \mathrm{~cm}^{2} / \mathrm{Vs}$, reasonably reflecting the high quality and the uniformity of the GSOS (Figure S10b). In addition, we employed thus-obtained GSOS as electrode pairs for demonstrating its potential for building nanoscale devices array. EBL was firstly used to draw forth the extended circuit on the GSOS electrodes, as schematically shown in Figure S10a. The randomly distributed $\mathrm{MoS}_{2}$ flakes were transferred onto the GSOS, thus we can measure the electrical property of the $\mathrm{MoS}_{2}$ as long as the flake can contact with a pair of graphene single crystal electrodes. The spacing of the GSOS can be controlled by adjusting the flow rate in the seed arranging process in order to match the typical size of the as-transferred $\mathrm{MoS}_{2}$ flake or any other materials. The design of the GSOS based electrode groups is similar to the quantifoil commonly used in the fundamental TEM characterizations, which can well achieve the location of the randomly distributed samples and facilitate the following characterization. The as-derived mobility of the $\mathrm{MoS}_{2}$ using GSOS as electrodes for achieving ohmic contact can be as high as 438 $\mathrm{cm}^{2} \mathrm{~V}^{-1} \mathrm{~s}^{-1}$, which is significantly higher than the reported results, ${ }^{6,7}$ as seen in Figure S10c and S10d, prefiguring a wide application of GSOS in the electronic devices.
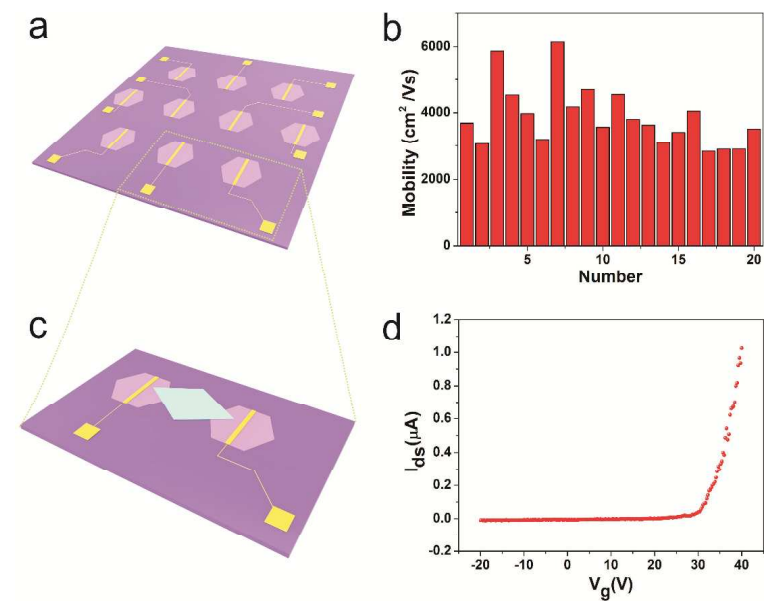

Figure S10. Electrical characteristics of FET devices based on GSOS. (a) Schematic of electrode pairs array based on GSOS. (b) Histogram of the mobility distribution for 20 devices. The mobility is $3882 \pm 896 \mathrm{~cm}^{2} \mathrm{~V}^{-1} \mathrm{~s}^{-1}$. (c) Schematic of a $\mathrm{MoS}_{2}$ device structure with bottom-graphene electrodes. (d) The transfer characteristics of the $\mathrm{MoS}_{2}$-based device in c. 
10. Electrical characteristics of FET devices based on the unassembled graphene single crystals

The mobility is $3729 \pm 665 \mathrm{~cm}^{2} \mathrm{~V}^{-1} \mathrm{~s}^{-1}$. The mobility of the assembled GSOS $\left(3882 \pm 896 \mathrm{~cm}^{2}\right.$ $\mathrm{V}^{-1} \mathrm{~s}^{-1}$, Figure $\left.\mathrm{S} 10 \mathrm{~b}\right)$ is comparable with the unassembled ones. It indicates that the self-assembly process will not damage the quality of the graphene single crystals. The high quality of the component in the GSOS will be well kept.

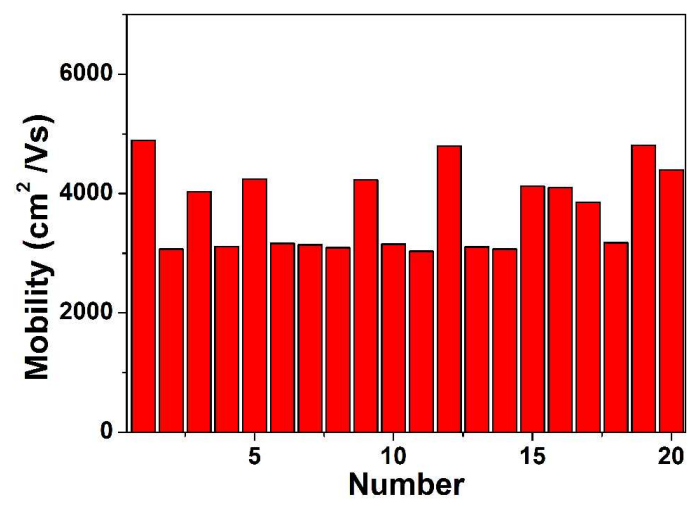

Figure S11. Histogram of the mobility distribution for 20 FET devices based on the unassembled graphene single crystals. 


\section{Reference}

1. Horcas, I.; Fernandez, R.; Gomez-Rodriguez, J. M.; Colchero, J.; Gomez-Herrero, J.; Baro, A. M. Rev. Sci. Instrum. 2007, 78, 013705.

2. Witten, T. A.; Sander, L. M. Phys. Rev. Lett. 1981, 47, 1400.

3. Wohner, N.; Lam, P. K.; Sattler, K. Carbon 2015, 82, 523.

4. Stewart, J. J. P. J. Comput. Chem. 1989, 10, 209.

5. Xue, Y.; Wu, B.; Jiang, L.; Guo, Y.; Huang, L.; Chen, J.; Tan, J.; Geng, D.; Luo, B.; Hu, W.; Yu, G.; Liu, Y. J. Am. Chem. Soc. 2012, 134, 11060.

6. Radisavljevic, B.; Radenovic, A.; Brivio, J.; Giacometti, V.; Kis, A. Nat. Nanotech. 2011, 6, 147.

7. Chen, W.; Zhao, J.; Zhang, J.; Gu, L.; Yang, Z.; Li, X.; Yu, H.; Zhu, X.; Yang, R.; Shi, D.; Lin, X.; Guo, J.; Bai, X.; Zhang, G. J. Am. Chem. Soc. 2015, 137, 15632. 